\title{
Analysis of mapping strategies for turbo-coded space-time block codes
}

\author{
Aydin Sezgin \\ Fraunhofer-Institute, HHI, Berlin, Germany \\ sezgin@hhi.de
}

Abstract - We propose a "turbo" coding scheme for the multiple-input multiple-output (MIMO) Rayleigh fading channel consisting of the serially concatenation of a block code as the outer code and different orthogonal space-time block codes (STBC) for more than two transmit antennas as the inner code. Here, we consider the orthogonal STBC as a mapping scheme in space and time. At the receiver, we apply iterative space-time detection and decoding. We analyze the impact of different mapping strategies on the information transfer of the soft-input-soft-output (SISO) space-time detector. Moreover, we analytically show that additional performance gains over Gray mapping can be obtained by different mapping strategies. Furthermore, we use extrinsic information transfer characteristics (EXITcharts) in order to predict the performance and the behavior of the system.

\section{INTRODUCTION}

In recent years, the goal of providing high speed wireless data services has generated a great amount of interest among the research community. Recent information theoretic results have demonstrated that the capacity of the channel in the presence of Rayleigh fading improves significantly by using multiple transmit and receive antennas [1], [2], [3]. More recently, the authors in [4], [5], [6] proposed to use a powerful channel code (e.g. turbo codes [7]) in concatenation with a space-time unitary matrix differential modulation code or orthogonal spacetime block codes (STBC) [8], [9] in order to achieve significant coding gains. Space-time block codes from orthogonal design provide the maximum possible diversity gain for a MIMO channel, but no coding gain.

In this work, we consider the application of channel codes as the outer code together with different orthogonal space-time block codes for more than two transmit antennas as the inner code in order to improve the performance of the uncoded system, and in order to achieve the capacity promised by the information theoretic results. Decoding at the receiver is done in an iterative manner between the SISO space-time detector and the SISO channel decoder. We consider different mapping strategies and analytically show that it is possible to improve the performance by employing other mapping schemes than Gray mapping. In addition to this, we analyze the space-time detection and decoding components with extrinsic information transfer (EXIT) charts. EXIT charts have been proposed in [10] as a quasi-analytical tool for predicting the convergence behavior and the performance of concatenated coding systems.

The remainder is organized as follows: In Section II, we introduce the system model, establish notation and briefly review the STBC scheme. The SISO receiver with the detecting and decoding components as well as analytical considerations
D. Wübben and V. Kühn

University of Bremen, Bremen, Germany

\{wuebben,kuehn\}@ant.uni-bremen.de

are described in Section III. Finally, some simulation results are given in Section IV, and our main conclusions are summarized in Section V.

\section{SYSTEM MODEL}

We consider a system with $n_{T} \geq 3$ transmit and $n_{R}$ receive antennas. We serially combine a STBC for different sizes of transmit antennas with a channel block code, namely an extended $\mathrm{BCH}(8,4)$ code to achieve low probability of error for small signal-to-noise-ratios (SNR). After the encoding and interleaving step the $F_{L}$ coded bits in the bit sequence $\left\{c_{1}, \ldots, c_{F_{L}}\right\}$, where $F_{L}$ denotes the frame length, are mapped onto symbols $s \in \mathcal{C}$ from a given constellation $\mathcal{C}$, e.g. $M$-PSK. The function $s=f(\mathbf{c})$ describes the mapping of $m=\log _{2}(M)$ consecutive bits contained in the vector $\mathbf{c}$ onto one constellation symbol $s$. The symbols $s$ are then space-time coded according to the $p \times n_{T}$ space-time generator matrix $\mathcal{G}_{n_{T}}$ or $\mathcal{H}_{n_{T}}$. In this work, we consider the performance of the space-time block codes with the generator matrices $\mathcal{G}_{3}, \mathcal{G}_{4}, \mathcal{H}_{3}$ and $\mathcal{H}_{4}$ from [9]. The code rate $R$ is given by $R=q / p$, where $q$ is the number of different symbols and $p$ is the number of time samples. Therefore, the code rate of $\mathcal{H}_{3}$ and $\mathcal{H}_{4}$ is $R=3 / 4$ and the code rate of $\mathcal{G}_{3}$ and $\mathcal{G}_{4}$ is $R=1 / 2$. Our system model is defined by

$$
r_{t}^{j}=\sum_{i=1}^{n_{T}} h_{i, j} s_{t}^{i}+n_{t}^{j},
$$

where $r_{t}^{j}$ is the received signal at time $t$ and receive antenna $j$, $s_{t}^{i}$ is the transmitted signal from a given constellation at time $t$ and transmit antenna $i, h_{i, j}$ is the complex flat fading channel path gain from transmit antenna $i$ to receive antenna $j$ and the noise $n_{t}^{j}$ is a complex Gaussian random variable at time $t$ and receive antenna $j$. The real and imaginary parts of $n_{t}^{j}$ are independent and Gaussian $\mathcal{N}\left(0, n_{T} /(2 \mathrm{SNR})\right)$ distributed.

Throughout the paper, we assume that the channel path gains are i.i.d. (identically independent distributed) and that successive blocks of channel realizations are independent. The effect of spatial correlation among antennas on the performance of space-time codes is studied in [11]. We further assume that perfect channel state information (CSI) is available at the receiver and that the transmitter has no CSI. The real and imaginary parts of the channel gains are independent and Gaussian distributed random variables with $\mathcal{N}(0,1 / 2)$ per dimension. The average energy of the symbols transmitted from each antenna is denoted as $E_{s}$, so that the average power of the received signal at each antenna is $n_{T} E_{s}$ and the signal-to-noiseratio is $E_{s} / N_{0}$.

\section{ITERATIVE DETECTION AND DECODING}

Fig. 1 shows the structure of the receiver which consists of two stages: the Space-Time Detector (STD) described in the following subsection B, and a Maximum A Posteriori (MAP) 
Decoder [12]. The two stages are separated by deinterleavers and interleavers. The receiver works as follows: In the first iteration, the switch in Fig. 1 is in position 1; assuming equally likely bits, the resulting a priori information $\lambda_{A, S T D}$ is zero. Now, the Space-Time Detector has to compute the extrinsic information $\lambda_{E, S T D}$ only from the observations of the channel output. The extrinsic information $\lambda_{E, S T D}$ is deinterleaved and fed into the MAP decoder as a priori information $\lambda_{A, M A P}$. Based on this a priori information and the trellis representation of the channel code [13], the MAP-Decoder computes the extrinsic information $\lambda_{E, M A P}$. After interleaving, this extrinsic information $\lambda_{E, M A P}$ is fed back to the STD as a priori information $\lambda_{A, S T D}$ for the following iterations. After the first iteration, the switch in Fig. 1 is in position 2.

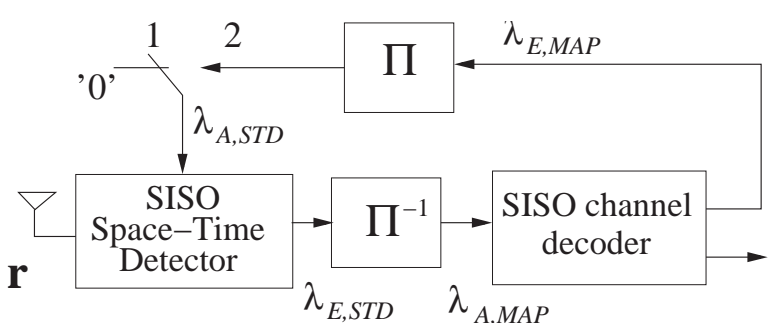

Fig. 1. Model of the SISO receiver with SISO Space-Time Detector, SISO channel decoder, interleaver and deinterleaver.

\section{A. Characterizing Different Mappings by Mutual Information}

In the following, we analyze the impact of different 8PSK mappings (comparable to the 8ASK mappings in [14]) on the transfer characteristics of the detector for an AWGN channel. The extension to a Rayleigh fading channel is straightforward. The mutual information between transmitted constellation symbol $s=f(\mathbf{c})$ and received AWGN channel output $r$ is given by (assuming that all constellation symbols are equiprobable)

$$
I(s ; r)=\frac{1}{M} \sum_{n=1}^{M} \int_{-\infty}^{\infty} p\left(r \mid s=s_{n}\right) \log _{2} \frac{p\left(r \mid s=s_{n}\right)}{p(r)} d r
$$

with conditional probability density function (PDF)

$$
p\left(r \mid s=s_{n}\right)=\frac{1}{\pi \sigma^{2}} \exp \left(-\frac{\left|r-s_{n}\right|^{2}}{\sigma^{2}}\right)
$$

and $p(r)=\frac{1}{M} \sum_{n=1}^{M} p\left(r \mid s=s_{n}\right)$, where $\log _{2}(\cdot)$ denotes the base $2 \operatorname{logarithm}, M=2^{m}$ and $\sigma^{2}$ is the noise variance. With the chain rule of mutual information it can be shown that the mutual information can be decomposed into $I(s ; r)=I(\mathbf{c} ; r)=I\left(c_{1}, \ldots, c_{m} ; r\right)=\sum_{L=0}^{m-1} I_{L}$, where $I_{L}$ is the average mutual information [14], when $L$ bits are already known to the receiver. Note that conditioning (i.e. increasing a priori knowledge) increases the mutual information, i.e. $I_{L} \geq I_{L-1}$. In Table 1 we show the mapping function $f(\mathbf{c})$ for five different mappings, which we choose from all possible 8PSK mappings. The constellation positions given in Table 1 are depicted in Fig. 2 for illustration.

\begin{tabular}{|c|c|c|c|c|c|}
\hline \multicolumn{5}{|c|}{ 8PSK Mappings } & constellation \\
position
\end{tabular}

TABLE 1

$f(\mathbf{c})$ FOR 5 DIFFERENT MAPPINGS WITH 8PSK

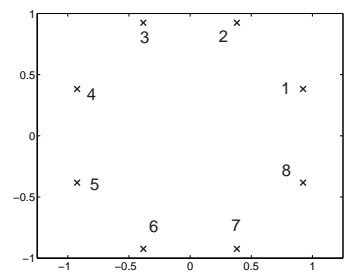

Fig. 2. 8PSK-constellation

Comparing the information transfer for different mappings in Table 2, we see that for Gray mapping the difference between $I_{2}$ and $I_{0}$ is not as large as in the case of the other mappings. This means that increasing a priori knowledge has only small impact on the information transfer of the detector for Gray mapping. Interestingly, this is not true for the other mappings. Here, we observe an improved information transfer by increasing the a priori knowledge. Additionally, $I_{0}$ is larger with Gray mapping in comparison to the other mappings. Therefore, we expect a better performance with Gray mapping in the case, where no a priori knowledge is available at the receiver.

\begin{tabular}{|c|r|r|r|c|}
\hline \multirow{2}{*}{$\begin{array}{c}\text { 8PSK } \\
\text { mappings }\end{array}$} & \multicolumn{3}{|c|}{$I_{L}$} & \multirow{2}{*}{$\sum I_{L}=I(s ; r)$} \\
\cline { 2 - 4 } & $I_{0}$ & $I_{1}$ & $I_{2}$ & \\
\hline Gray & 0.7805 & 0.7819 & 0.7830 & 2.345 \\
\hline natural & 0.6369 & 0.8265 & 0.8819 & 2.345 \\
\hline "d21" & 0.6321 & 0.7736 & 0.9395 & 2.345 \\
\hline "d23" & 0.5380 & 0.8182 & 0.9889 & 2.345 \\
\hline anti Gray & 0.4933 & 0.8723 & 0.9796 & 2.345 \\
\hline
\end{tabular}

TABLE 2

CONDITIONAL MUTUAL INFORMATION $I_{L}$ FOR DIFFERENT 8PSK MAPPINGS AT $E_{b} / N_{0}=6 \mathrm{~dB}$

\section{B. Space-Time Detector with Soft Outputs}

In the following, we focus without loss of generality on the first $p$ consecutive channel uses. The space-time detector at the receiver computes the log-likelihood ratios of the coded bits $\left\{c_{k}\right\}_{k=1}^{q m}$ corresponding to the transmitted sequence $\mathbf{s}=$ $\left\{s_{1} \ldots, s_{q}\right\}$ from the received sequence $\left\{\mathbf{r}_{1}, \ldots, \mathbf{r}_{p}\right\}$, where $\mathbf{r}_{t}=\left[r_{t}^{1}, \ldots, r_{t}^{n_{R}}\right]$, with

$$
\Lambda_{\mathrm{STD}}\left(c_{k}\right)=\log \frac{\sum_{\mathbf{s} \in \mathcal{S}^{+}} P\left[\mathbf{r}_{1}, \ldots, \mathbf{r}_{p} \mid \mathbf{s}\right] \cdot P[\mathbf{s}]}{\sum_{\mathbf{s} \in \mathcal{S}^{-}} P\left[\mathbf{r}_{1}, \ldots, \mathbf{r}_{p} \mid \mathbf{s}\right] \cdot P[\mathbf{s}]} .
$$


In (4), $\log (\cdot)$ denotes the natural logarithm, $\mathcal{S}^{+}=\left\{\mathbf{s}: s_{i}=\right.$ $\left.f\left(\mathbf{c}_{i}\right), c_{k}=0\right\}$ is the set of transmitted sequences such that $c_{k}=0$ and $\mathcal{S}^{-}=\left\{\mathbf{s}: s_{i}=f\left(\mathbf{c}_{i}\right), c_{k}=1\right\}$ is the set of transmitted sequences such that $c_{k}=1$. Since we have a sufficiently long pseudo-random bit interleaver, we may assume that the probabilities $P\left[c_{l}\right]$ of the coded bits $c_{l}$ are independent. Thus, we have $P[\mathbf{s}]=\prod_{l=1}^{q m} P\left[c_{l}\right]$. After some manipulations we obtain

$$
\begin{aligned}
& \Lambda_{\mathrm{STD}}\left(c_{k}\right)= \\
& \log \frac{\sum_{\mathbf{s} \in \mathcal{S}^{+}} \exp \left(\frac{-\sum_{t=1}^{p} \sum_{j=1}^{n_{R}}\left|r_{t}^{j}-\sum_{i=1}^{n_{T}} h_{i, j} s_{t}^{i}\right|^{2}}{\sigma^{2}}\right) \prod_{l=1}^{q m} P\left[c_{l}\right]}{\sum_{\mathbf{s} \in \mathcal{S}^{-}} \exp \left(\frac{-\sum_{t=1}^{p} \sum_{j=1}^{n_{R}}\left|r_{t}^{j}-\sum_{i=1}^{n_{T}} h_{i, j} s_{t}^{i}\right|^{2}}{\sigma^{2}}\right) \prod_{l=1}^{q m} P\left[c_{l}\right]}
\end{aligned}
$$

holds. The probabilities $P\left[c_{k}=0\right]$ and $P\left[c_{k}=1\right]$ are present in every term of the summation in the numerator and denominator, respectively. Therefore, we can write (5) as

$$
\begin{aligned}
& \Lambda_{\mathrm{STD}}\left(c_{k}\right)= \\
& \underbrace{\log \frac{\sum_{\mathbf{s} \in \mathcal{S}^{+}} \exp \left(-\frac{\sum_{t=1}^{p} \sum_{j=1}^{n_{R}}\left|r_{t}^{j}-\sum_{i=1}^{n_{T}} h_{i, j} s_{t}^{i}\right|^{2}}{\sigma^{2}}\right) \prod_{\substack{l=1, l \neq k}}^{q m} P\left[c_{l}\right]}{\sum_{\mathbf{s} \in \mathcal{S}^{-}} \exp \left(-\frac{\sum_{t=1}^{p} \sum_{j=1}^{n_{R}}\left|r_{t}^{j}-\sum_{i=1}^{n_{T}} h_{i, j} s_{t}^{i}\right|^{2}}{\sigma^{2}}\right) \prod_{\substack{l=1, l \neq k}}^{q m} P\left[c_{l}\right]}}_{\text {extrinsic information } \lambda_{E, S T D}} \\
& +\underbrace{\log \frac{P\left[c_{k}=0\right]}{P\left[c_{k}=1\right]}}_{\text {a priori information } \lambda_{A, S T D}} .
\end{aligned}
$$

The log-likelihood ratio $\Lambda_{\mathrm{STD}}$ for $c_{k}$ is now decomposed into two parts $\Lambda_{\mathrm{STD}}=\lambda_{E, S T D}+\lambda_{A, S T D}$, where $\lambda_{A, S T D}$ is the a priori information obtained through the iterative decoding process and $\lambda_{E, S T D}$ is obtained from the observations of the channel output. We can now apply this result to the space-time block codes $\mathcal{G}_{3}, \mathcal{H}_{3}, \mathcal{G}_{4}$ and $\mathcal{H}_{4}$. As an example, we do this for $\mathcal{G}_{3}$. Since the columns of the generator matrix of the spacetime block code $\mathcal{G}_{3}$ are orthogonal to each other, we can decompose (6) into four parts, where each part is only a function of $s_{i}$ with $i=1 \ldots 4$, given as

$$
\begin{array}{r}
\Lambda_{\mathrm{STD}}\left(c_{k}\right)= \\
\log \frac{\sum_{s_{i} \in \mathcal{S}_{i}^{+}} \exp \left(-\frac{\left|\tilde{r}_{i}-s_{i}\right|^{2}+\tilde{h}\left|s_{i}\right|^{2}}{\sigma^{2}}\right) \prod_{\substack{l=1, l \neq k}}^{m} P\left[c_{l}\right]}{\sum_{s_{i} \in \mathcal{S}_{i}^{-}} \exp \left(-\frac{\left|\tilde{r}_{i}-s_{i}\right|^{2}+\tilde{h}\left|s_{i}\right|^{2}}{\sigma^{2}}\right) \prod_{\substack{l=1 \\
l \neq k}}^{m} P\left[c_{l}\right]} \\
+\log \frac{P\left[c_{k}=0\right]}{P\left[c_{k}=1\right]} \quad \text { for }(i-1) m<k \leq i m .
\end{array}
$$

The variables $\tilde{h}$ and $\tilde{r}_{1}, \cdots, \tilde{r}_{4}$ are given according to [15, p. 458]. $\mathcal{S}_{i}^{+}=\left\{s_{i}: c_{k}=0\right\}$ is the set of space-time symbols such that $c_{k}=0$ and $\mathcal{S}_{i}^{-}=\left\{s_{i}: c_{k}=1\right\}$ is the set of space-time symbols such that $c_{k}=1$ for any $k$ with $(i-1) m<k \leq i m$. This dramatically reduces the complexity of the SISO space-time detector.

The code bit probabilities $P\left[c_{k}\right]$ in (7) can be expressed in terms of their a priori information as

$$
P\left[c_{k}\right]= \begin{cases}\frac{1}{1+\exp \left(+\lambda_{A, S T D}\right)} & \text { for } c_{k}=1 \\ \frac{1}{1+\exp \left(-\lambda_{A, S T D}\right)} & \text { for } c_{k}=0\end{cases}
$$

It is possible to simplify the computational complexity for $\Lambda_{\mathrm{STD}}$ in (7), by using the following approximation

$$
\begin{aligned}
\Lambda_{\mathrm{STD}}\left(c_{k}\right) \approx & \max _{s_{i} \in \mathcal{S}_{i}^{+}} \log P\left[\mathbf{r}_{1}, \ldots, \mathbf{r}_{p} \mid s_{i}\right] P\left[s_{i}\right] \\
& -\max _{s_{i} \in \mathcal{S}_{i}^{-}} \log P\left[\mathbf{r}_{1}, \ldots, \mathbf{r}_{p} \mid s_{i}\right] P\left[s_{i}\right],
\end{aligned}
$$

which we used in our simulations in the next section due to numerical stability. In order to perform the soft decoding at the next stage of the receiver (i.e. the MAP decoder), we regard these log-likelihoods as the observations from a BPSK modulation over an additive white Gaussian noise channel as described in [16].

\section{SiMULATION RESULTS}

In this section, the simulation results of the proposed scheme and their interpretation are presented. Fig. 3 shows the extrinsic information transfer characteristics of the block code with outer rate $R_{\text {out }}=1 / 2$. Additionally, the curves of the space-

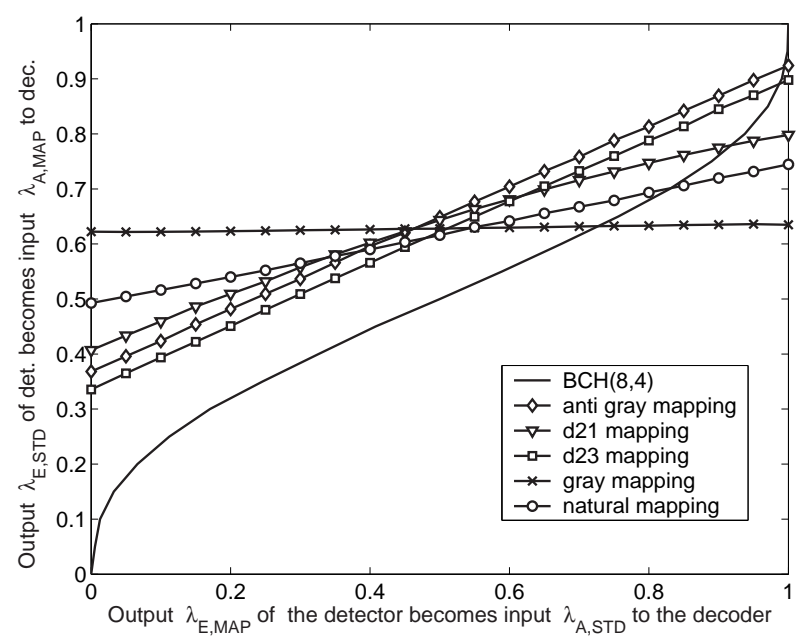

Fig. 3. $\mathrm{SNR}=1 \mathrm{~dB}$, EXIT charts of outer rate $R_{\text {out }}=1 / 2$ decoder and transfer characteristics of inner detector $\mathcal{G}_{3}$. We choose the SNR value such that the "turbo-cliff"-region appears in the Figure.

time detector for $\mathcal{G}_{3}$ as the inner decoder for different mapping strategies are depicted. Due to lack of space, we omit here the curves for $\mathcal{H}_{3}, \mathcal{G}_{4}$ and $\mathcal{H}_{4}$. Different mappings result in transfer characteristics of different slopes. It is important to know that for the EXIT chart predictions on code performance we assumed very large interleavers and a fast fading channel, in which the channel is selected independently for each spacetime code matrix (i.e. channel is constant for only $p$ channel uses). However, in practice using large interleavers is not applicable. In our bit error rate simulations later on in this section 
we use moderate interleaver sizes. Due to this both assumptions the EXIT charts can be regarded only as asymptotic results. Therefore, the "turbo-cliff"-region occurs not exactly at the predicted SNR values. Note that the detector transfer characteristics are almost straight lines and that at low SNR values increasing the SNR just shifts the curve up. Additionally, note that for high SNR values, the slope of the detector transfer characteristics is also affected (not depicted here). From the figure, we see also that the natural mapping provides good extrinsic output at the beginning but provides diminishing output for higher a priori input $\lambda_{A}$. For the anti gray mapping it is the other way around. The detector which uses gray mapping provides almost the same extrinsic output for all a priori input, which confirms the results from Table 2 in section A. Therefore, we expect that the performance of the detector with gray and natural mapping is good in the low SNR regime and for a few iterations in comparison to the other mappings. But in the high SNR regime and for more iterations, we expect that it is the other way around. Note that the axes are swapped for the outer code: $\lambda_{A}$ is on the ordinate, $\lambda_{E}$ on the abscissa.

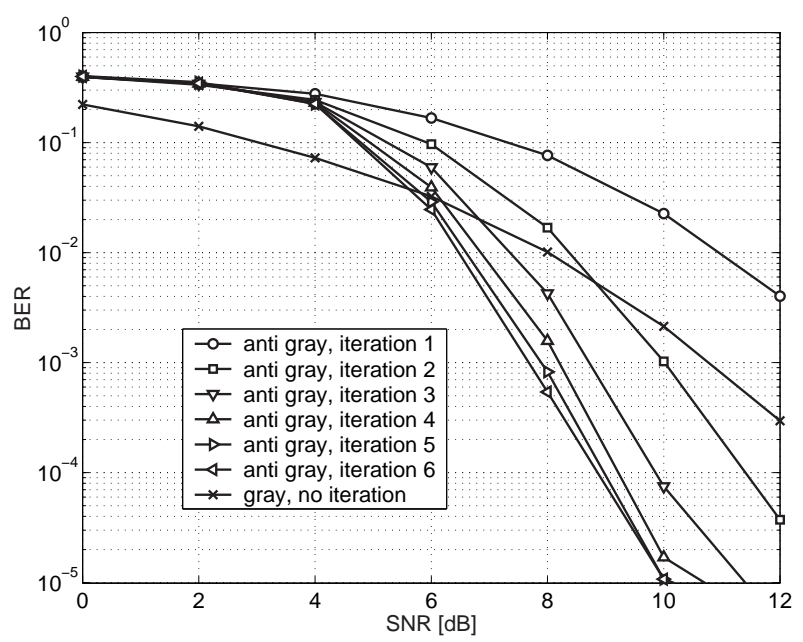

Fig. 4. Performance of the considered outer code (extended $\mathrm{BCH}(8,4)$ ) in concatenation with $\mathcal{G}_{3}$ with different mapping strategies, $n_{T}=3$ transmit and $n_{R}=1$ receive antenna, 8PSK modulation.

For verification of the observations from the EXIT-Charts, we present the bit error rates (BERs) for gray and anti gray mapping in Fig. 4 for $\mathcal{G}_{3}$. Since the transfer characteristics of all other mapping strategies are almost always between the transfer characteristics of gray and anti gray mapping, only their BERs are depicted. Coding is performed over multiple block fading channels. The transmitted bits are organized in frames of length $F_{L}=432$. We assume that one block fading channel is constant for $\tau=24$ channel uses. We observe that although the curves are relatively flat at iteration 1 , there is a significant improvement with further iterations. However, in the case of gray mapping iteratively decoding yields negligible gain, so only the first iteration is plotted. Furthermore, simulation results show, that there occurs a saturation of the improvement for the other mappings beyond iteration 6 . This behavior can be explained by the fact that after each iteration the extrinsic output of the receiver components tends to a Gaussian distribution according to the central limit theorem but the correlation between the extrinsic information and the channel output increases after each iteration [13]. Hence, the improvement of the BER-performance diminishes after each iteration. As predicted in the EXIT-Charts, the BER-performance of the gray mapping is better than the BER-performance of the anti gray mapping in the low SNR regime. In the high SNR regime, however, it is the other way around. Note that this performance difference to gray mapping can further enhanced with a larger frame length or a smaller $\tau$. If we compare this results with the uncoded system with gray mapping in [15, Fig. 4] and coded system with anti gray and gray mapping, we see that there is a significant performance gain compared to the uncoded system.

\section{CONCLUSION}

In this paper, the serial concatenation of a block code, namely an extended $\mathrm{BCH}(8,4)$ code, with different space-time block codes has been proposed. We analyzed different mapping strategies on the information transfer of the SISO detector and analytically showed that additional performance gain is achieved in comparison to Gray mapping. The performance of this scheme was investigated with EXIT-charts and BERs and compared with the uncoded scheme in [15]. Simulation results show that this scheme performs significantly better in terms of bit error rates in comparison to the uncoded system.

\section{REFERENCES}

[1] E. Telatar, "Capacity of multi-antenna gaussian channels," European Trans. on Telecomm. ETT, vol. 10, no. 6, pp. 585-596, November 1999.

[2] G.J. Foschini and M.J. Gans, "On limits of wireless communications in a fading environment when using multiple antennas," Wireless Personal Communications, vol. 6, no. 3, pp. 311-335, March 1998.

[3] E. Biglieri, G. Caire, and G. Taricco, "Limiting performance of blockfading channels with multiple antennas," IEEE Trans. on Info. Theory, vol. 47, no. 4, pp. 1273-1289, May 2001.

[4] G. Bauch, "Concatenation of space-time block codes and Turbo-TCM," IEEE Int. Conf. on Communications, Vancouver, BC, Canada, vol. 2, pp. $1202-1206,6-10$ June 1999.

[5] T.H. Liew and L. Hanzo, "Space-time codes and concatenated channel codes for wireless communications," Proceedings of the IEEE, vol. 90, pp. $187-219$, February 2002.

[6] A. Steiner, M. Peleg, and S. Shamai (Shitz), "Iterative decoding of spacetime differentially coded unitary matrix modulation," IEEE Trans. on Signal Processing, vol. 50, no. 10, pp. 2385-2395, October 2002.

[7] C. Berrou and A. Glavieux, "Near optimum error correcting coding and decoding: turbo codes," IEEE Trans. on Communications, vol. 44, no. 10, pp. 1261-1271, October 1996.

[8] S.M. Alamouti, "A simple transmitter diversity scheme for wireless communications," IEEE Journal on Selected Areas in Communications, vol. SAC-16, pp. 1451-1458, October 1998.

[9] V. Tarokh, H. Jafarkhani, and A.R. Calderbank, "Space-time block code from orthogonal designs," IEEE Trans. on Info. Theory, vol. 45, no. 5, pp. 1456-1467, July 1999.

[10] S. ten Brink, "Convergence of iterative decoding," IEE Electron. Lett., vol. 35, no. 10, pp. 806-808, May 1999.

[11] M. Uysal and C.N. Georghiades, "Effect on spatial correlation on performance of space-time codes," IEE Elec. Letters, vol. 37, no. 3, pp. 181-183, February 2001.

[12] L. Bahl, J. Cocke, F. Jelinek, and J. Raviv, "Optimal decoding of linear codes for minimizing symbol error rate," IEEE Trans. on Information Theory, vol. 20, pp. 284-287, March 1974.

[13] J. Hagenauer, E. Offer, and L. Papke, "Iterative decoding of binary and convolutional codes," IEEE Trans. on Information Theory, vol. 42, pp. 429-445, March 1996.

[14] S. ten Brink, "Designing iterative decoding schemes with the extrinsic information transfer chart," AEUE Intern. Journal of Electr. and Commun., vol. 54, pp. $187-219,2000$.

[15] V. Tarokh, H. Jafarkhani, and A.R. Calderbank, "Space-time block coding for wireless communications: Performance results," IEEE Journal on Sel. Areas in Communications, vol. 17, no. 3, pp. 451 -460, March 1999.

[16] T.M. Duman, Turbo codes and turbo coded modulation systems: Analysis and performance bounds, Ph.D. thesis, Northeastern University, Dept. of Electrical and Computer Engineering, Boston, MA, 1998. 